\title{
Electrochemical Characteristics of DNA Modified Electrode as a Function of Percent Binding
}

Rahul Tevatia, ${ }^{[a]}$ Abhijeet Prasad, ${ }^{[b]}$ Ravi F. Saraf ${ }^{\star[b],[c]}$

[a] Vajra Instruments, Inc, Lincoln, NE 68512

[b] Department of Chemical and Biomolecular Engineering, University of NebraskaLincoln, NE 68512;

[c] Nebraska Center for Material and Nanoscience, University of Nebraska-Lincoln, NE 68512

*rsaraf2@unl.edu

SI Table of Contents:

S1. Chemicals and DNA handling S-2

S2. Chip Fabrication, Spotting, EREB and MCH Backfilling S-2

S3. qPCR Analysis for Probe Coverage and Fraction Target Binding S-6

S4. The Analysis of SEED Optics $\quad$ S-7

S5. Figures S7 to S16 $\quad$ S-11

S6. Fluorescence Analysis for Probe Coverage S-15

$\begin{array}{lr}\text { S7. Differential Pulse Voltammetry (DPV) } & \text { S-17 }\end{array}$

Figure S1, S2 S-3

Figure S3, S4 S-5

Figure S5 S-6

Figure $\mathbf{S 6} \quad$ S-7

Figure $\mathrm{S7} \quad \mathrm{S}-11$

Figure S8, S9, S10 S-12

Figure S11, S12 $\quad \mathrm{S}-13$

Figure S13, S14 $\quad$ S-14

Figure S15, S16 $\quad$ S-15

Figure $\mathrm{S} 17 \quad \mathrm{~S}-16$

$\begin{array}{lr}\text { Figure S18 } & \mathrm{S}-17\end{array}$

$\begin{array}{lr}\text { Figure S19 } & \text { S-18 }\end{array}$

$\begin{array}{lr}\text { Table S1 } & \text { S-17 }\end{array}$

$\begin{array}{lr}\text { References } & \text { S-18 }\end{array}$ 


\section{S1. Chemicals and DNA handling}

The photoresist SU8 and developer were purchased from Microchem. Acetone, ethanol, hydrogen peroxide $(40 \% \mathrm{v} / \mathrm{v})$, concentrated sulfuric acid, and cyclopentanone were chemical grade and purchased from Sigma-Aldrich. Tris(2-carboxyethyl)phosphine hydrochloride (TCEP), 1-mercapto-6-hexanol $(\mathrm{MCH})$, potassium ferrocyanide, and methylene blue (MB) were purchased from Sigma-Aldrich. Reference electrodes required a silver wire, reference filling, and reference electrode storage solutions (Sigma-Aldrich). Phosphate monobasic and phosphate dibasic (Sigma-Aldrich) were used to prepare $1.0 \mathrm{M}$ stock solution of phosphate buffer (the amount was calculated using the Henderson-Hasselbalch equation for a final $\mathrm{pH}=7.6$ ). Reagents for PCR, SYBR $^{\circledR}$ Green miRNA assay were purchased from Clontech Lab Inc. DNA/RNA probes and targets with or without thiol or Cy3 modifications were synthesized by Integrated DNA Technologies, Inc (IDT).

All DNA/RNA were received in lyophilized form and substituted with DNase/Rnase-free water to $1 \mathrm{mM}$ final concentration. These stocks were stored at $-20^{\circ} \mathrm{C}$. From $1 \mathrm{mM}$ master stocks, $10 \mu \mathrm{M}$ processed probes or unmodified targets were aliquot in $10 \mu \mathrm{l}$ and stored at $-20^{\circ} \mathrm{C}$. Each $10-\mu$ vial was used once to avoid any potential damage to the nucleic acids caused by repeated freeze-thaw cycles.

\section{S2. Chip Fabrication, Spotting, EREB and MCH Backfilling}

There were four steps to make the DNA electrode in an array of seventy-two $50 \mu \mathrm{m}$ diameter microwells by spotting followed by binding and backfilling steps (Figure 2(b)). First, $50-\mu \mathrm{m}$ diameter microwells were patterned by photolithography using $500-\mathrm{nm}$ thick SU8 photoresist (Figure S1 and S2(a)). Each of the five electrodes had a matrix of $6 \times 12$ microwells, while the two small electrodes had three microwells each. Second, the chip was exposed to oxygen $\left(\mathrm{O}_{2}\right)$ plasma (at 500 mTorr, $65 \mathrm{~W}$ ) for $60 \mathrm{sec}$ to clean the remaining SU8; and sSDNA probes were spotted on each individual microwell using a home-built spotter (Figure S2(b) and S3(a)). Third, the binding was performed by applying a potential between the electrode and the solution containing the target, miR155 (Figure S2(c)). The five large electrodes were subjected to the probe-target binding process, while no potential to bind was applied to the smaller electrodes. Thus, the microwells on the smaller electrode served as controls where no binding occurred. Fourth, the exposed Au electrode was backfilled with $\mathrm{MCH}$ (Figure S2(d)). As described, the binding was performed electrochemically and backfilling was a two-step process to ensure complete passivation of the electrode surrounding the DNA. Each of the four steps is discussed below. 


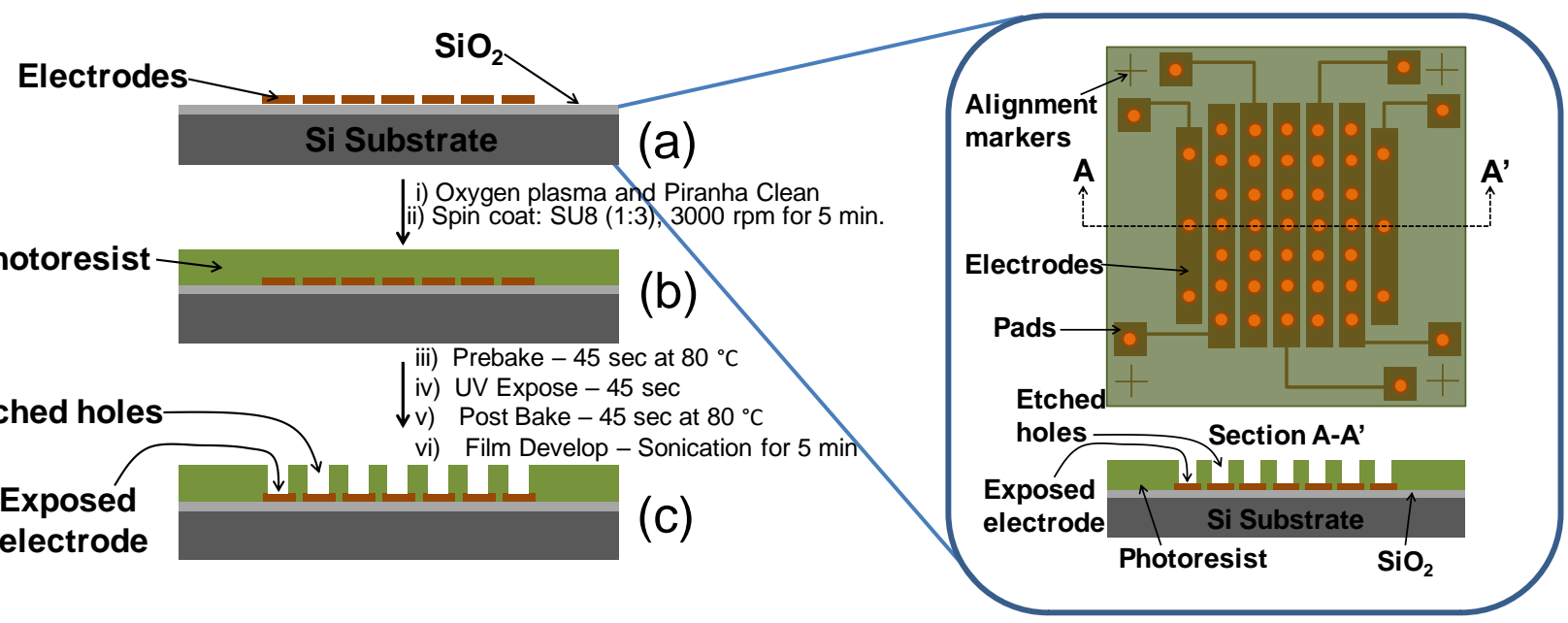

Figure S1: Cross-sectional view of the chip defined by A-A' in Figure 2. Broadly, the photolithography had the following steps: (a) initial chip with electrodes; (b) after spin coating SU-8 photoresist; and (c) the chip after exposure to UV light and development to obtain a pattern of etched holes. Right: Schematic of the chip with electrodes, connecting pads and microwells. For the study there are 3 and 72 microwells on the 2 control and 5 active electrodes.

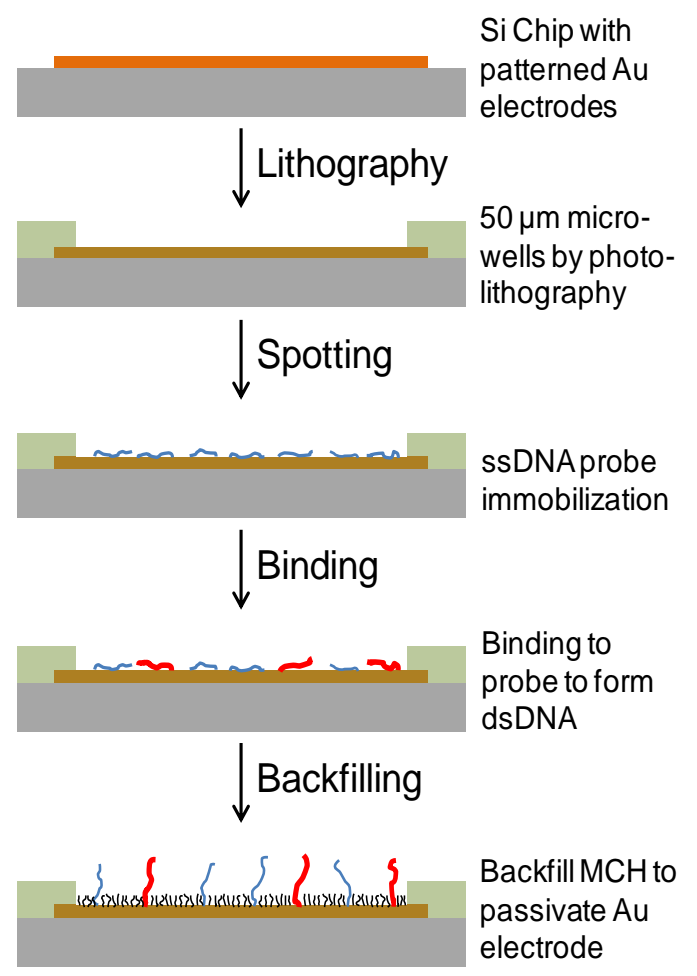

Figure S2: The four steps used to immobilize DNA on a typical microwell. 
a) Step 1: Chip fabrication - The microarray was made on a 1.2 by $1.2 \mathrm{~cm}$ silicon (Si) chip with $\sim 200 \mathrm{~nm}$ silicon dioxide $\left(\mathrm{SiO}_{2}\right)$ (thermal oxide) to provide an insulating surface. Gold $(\mathrm{Au})$ electrodes and accompanying circuitry for power and signal on the chip were patterned at the wafer level using standard photolithography followed by dicing of the wafer to make chips. The electrodes were $40 \mathrm{~nm}$ Au on $10 \mathrm{~nm}$ titanium (Ti) on silica. The Ti was used to promote adhesion between the silica and Au. The chip was coated with SU8 photoresist to make 72 and 3 holes of $50 \mu \mathrm{m}$ diameter on the longer and shorter electrodes, respectively, using a standard photolithography process (Figure S1). Briefly, the chip was cleaned in acetone, water, and ethanol followed by oxygen $\left(\mathrm{O}_{2}\right)$ plasma and subsequently immersed in piranha solution for $\sim 60 \mathrm{sec}$. The chip was dried by blowing clean nitrogen $\left(\mathrm{N}_{2}\right)$ through a $0.2 \mu \mathrm{m}$ Millipore ${ }^{\mathrm{TM}}$ filter. Photoresist SU8 (MicroChem 2025) was diluted by adding three times the volume of cyclopentanone which was spin cast onto the chip at 3,000 rpm for $60 \mathrm{sec}$ to produce a $~ 500-\mathrm{nm}$ thick film. The chip was prebaked for $45 \mathrm{~s}$ at $80^{\circ} \mathrm{C}$. The film was exposed to xenon $(\mathrm{Xe})$ light $(300 \mathrm{~W})$ for $45 \mathrm{~s}$ through a contact mask with a chromium $(\mathrm{Cr})$ metalized pattern on quartz. The chip was post-baked for $45 \mathrm{~s}$ at $80^{\circ} \mathrm{C}$ and developed in MicroChem SU8 developer in a sonicator for 5 minutes. The chip was washed with water/isopropanol for $\sim 60 \mathrm{~s}$ and hard baked at $180^{\circ} \mathrm{C}$ for $1 \mathrm{hr}$. The resulting pattern on each electrode was a microarray of $50 \mu \mathrm{m}$ holes exposing the underlying Au electrode

b) Step 2: Probe Spotting - After photolithography, the chip was exposed to $\mathrm{O}_{2}$ plasma to clean the leftover organics and expose the electrode in the microwell. The chip was then hard baked at $180^{\circ} \mathrm{C}$ to improve the adhesion and make the SU8 (back to being) hydrophobic. Individual solutions of the probes P155 and P21, were prepared in a volumetric ratio of one part of $1 \mathrm{mM}$ ssDNA probe and five parts of $500 \mathrm{mM}$ TCEP solution at $\mathrm{pH}$ 7. The solution was mixed and incubated at $25^{\circ} \mathrm{C}$ for 1 hour. The solution was subsequently diluted with $1 \mathrm{M} \mathrm{PB}$ at $\mathrm{pH} 7.6$ to obtain a final concentration of $2.5 \mu \mathrm{M}$ probe. The TCEP was utilized to cleave the disulfide linkages between the thio-groups among the probe molecules. The thiolated probe solution was spotted on each microwell using a $20 \mu \mathrm{m}$ capillary pin (Arrayit ${ }^{\circledR} 20 \mu \mathrm{m}$ tip) with the sample on a computer-controlled motorized $X-Y$ stage arm and machine vision (see Figure S3(a)). The drop was released by surface tension on the hydrophilic microwell. The drop was confined to the microwell owing to the hydrophobic nature of surrounding SU8 resist (see Figure S3(b)). The probe immobilization was allowed for 2 hours. The chip was cooled to $10^{\circ} \mathrm{C}$ and maintained in a humid environment to avoid evaporation of the approximate $100 \mathrm{~nL}$ drop during the immobilization process. Throughout the study on the short (control) electrode, the three microwells had no probe (i.e., blank), P155, and P21, respectively. The other five (active) electrodes had all of the 72 microwells immobilized with P155. 
Optical microscopy image

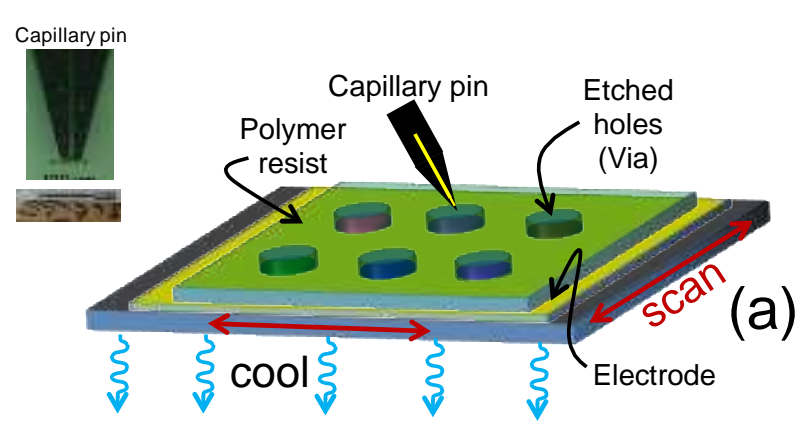

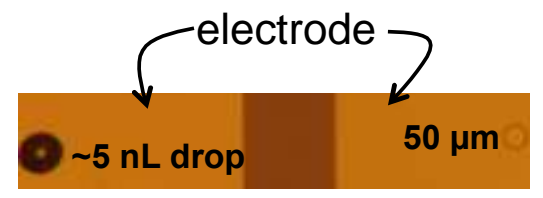

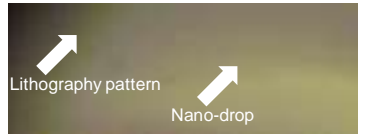

(b)

Figure S3: DNA probe immobilization (a) The schematic of the spotting. The inset is the capillary pin used to dispense the solution. (b) An optical image of the ssDNA probe solution droplet confined to the microwell. The drop was held by surface tension.

c) Step 3: Electrochemical Redox Enhanced Binding (EREB) - The binding was performed electrochemically using a potentiostat (Autolab, Metrohm) in a cyclic voltammetry (CV) setup on each of the five larger electrodes using a silver/ silver chloride $(\mathrm{Ag} / \mathrm{AgCl})$ reference electrode (RE) (Figure S4). The CV ramp was from -0.4 to $+0.3 \mathrm{~V}$ at a rate of, $\mathrm{s}=0.05 \mathrm{~V} / \mathrm{s}$. The solution had synthetic miR-155 target of concentration [c], in $100 \mathrm{mM} \mathrm{PB}\left(\mathrm{pH} \mathrm{7.6)}\right.$ with $50 \mathrm{mM} \mathrm{K}{ }_{4}\left[\mathrm{Fe}(\mathrm{CN})_{6}\right]$. The amount of binding was regulated in two ways: (i) [c]; and (ii) number of CV ramp cycles, $\mathrm{N}_{\mathrm{cv}}$. For a low amount of binding, [c] was fixed at $100 \mathrm{fM}$; and Ncv during EREB was varied from 2 to 28 cycles. For a high amount of binding, Ncv was constant at 32, while [c] was varied from $10^{2}$ to $10^{3} \mathrm{fM}$. As the electrochemical binding in this study was $<15$ min (at maximum $\mathrm{N}_{\mathrm{cv}}=32$ cycles), compared to $\sim 18 \mathrm{hrs}$ for conventional process by diffusion, there was no significant binding at $\mathrm{N}_{\mathrm{cv}}=0$. Thus, EREB on each active electrode can be considered to be an isolated experiment. For small control electrodes, $\mathrm{N}_{\mathrm{cv}}=0$. As a result, five EREB conditions were measured for each chip. The volume of the solution for EREB was $500 \mu \mathrm{L}$. The chamber was disassembled and washed after each EREB cycle.

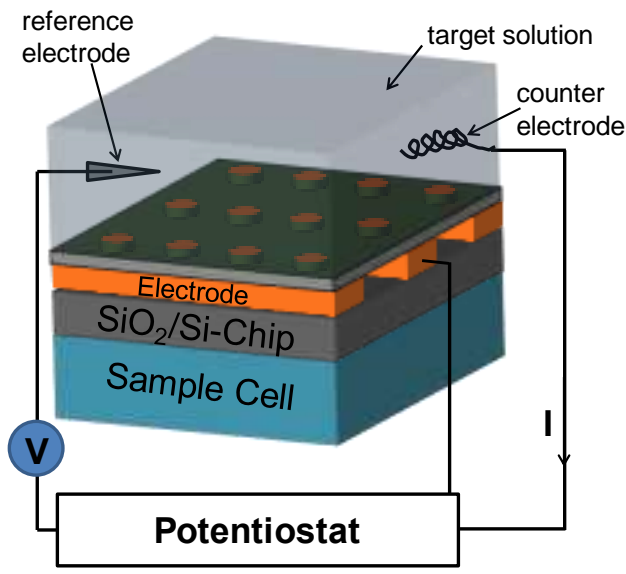

Figure S4: A schematic of the three-electrode setup for EREB process. The reference electrode was silver/silver chloride $(\mathrm{Ag} / \mathrm{AgCl})$, and the counter electrode was platinum $(\mathrm{Pt})$ wire. $\mathrm{CV}$ between one of the five "active electrode" and solution was applied for a fixed number of cycles, $\mathrm{N}_{\mathrm{CV}}$. 
d) Step 4: MCH backfill - Backfilling was one of the critical aspects of the fabrication. The $\mathrm{MCH}$ immobilization (i.e., backfilling) was performed in two steps. The chip was exposed to vapors from a $0.5-\mathrm{mL}$ solution of $10 \mathrm{mM} \mathrm{MCH}$ in DNAse/RNAse-free water at $37^{\circ} \mathrm{C}$ for $10 \mathrm{hr}$. The chip was dry with no condensation of water during the process. After rinsing in autoclaved deionized (DI) water, the chip was then immersed in a $2 \mathrm{~mL}$ solution of $10 \mathrm{mM} \mathrm{MCH}$ in $30 \% \mathrm{HPLC}$ grade ethanol for 3 hours with vigorous shaking at $100 \mathrm{rpm}$ in an incubator at $37^{\circ} \mathrm{C}$. The second incubation step is critical to plug the small holes in the $\mathrm{MCH}$ layer. The chip was subsequently rinsed and immersed in solution for SEED measurement.

\section{S3. qPCR Analysis for Probe Coverage and Fraction Target Binding}

The SYBR ${ }^{\circledR}$ Green method (Clontech Lab., Inc) was adopted to perform the cDNA synthesis and quantitative polymerase chain reaction (qPCR) measurements (QuantStudio $^{\text {TM }} 3$ RT-PCR, ABI, USA). Briefly, $3.75 \mu \mathrm{L}$ of a standard solution with a known concentration was added to reverse a transcriptase buffer and enzyme (the final reaction volume was $10 \mu \mathrm{L}$ ). The reverse transcriptase reaction was allowed to incubate at $37{ }^{\circ} \mathrm{C}$ for one hour, followed by denaturation of the RT enzyme at $85{ }^{\circ} \mathrm{C}$ for five minutes. The synthesized cDNA was diluted by 10 -fold. A $0.8 \mu \mathrm{L}$ portion was added to the master stock (SYBR Advantage Premix, ROX, miRNA-specific 5' and 3' primers) resulting in the final volume of $10 \mu \mathrm{L}$. Melting curves on qPCR products were also generated to confirm the specificity of the amplification. After qPCR, the data was analyzed while setting the threshold fluorescence to a constant value of 0.059 arbitrary units. The threshold was set to a constant for biological replicates. Based on Ct values, the number of probes or targets was calculated from the standard curve.

Figure S5 shows the standard curve for both the probe and target measure from known amount of synthetic ssDNA molecules in the RT-mixture.

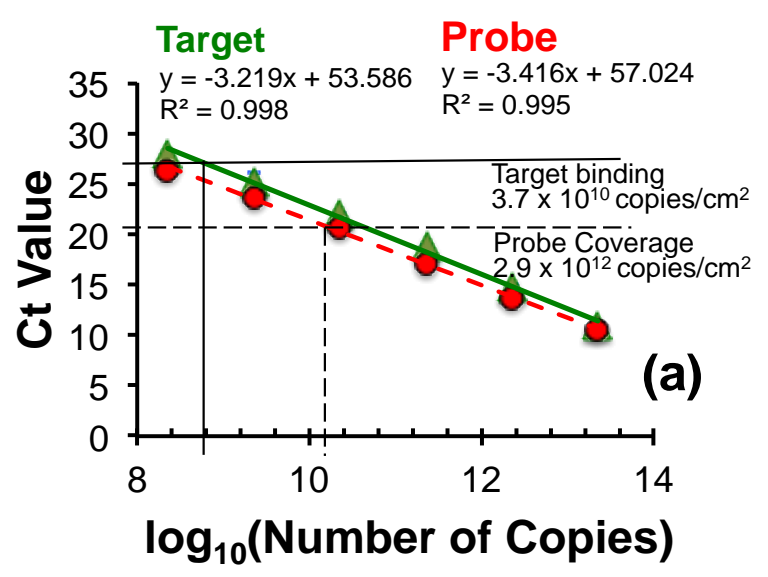

Figure S5: The standard curve of the probe and target for SYBRGreen qPCR. The copy number is the number of molecules in the RT mix for cDNA conversion. Below $10^{6}$ number of copies, the CT value the curve becomes nonlinear. 

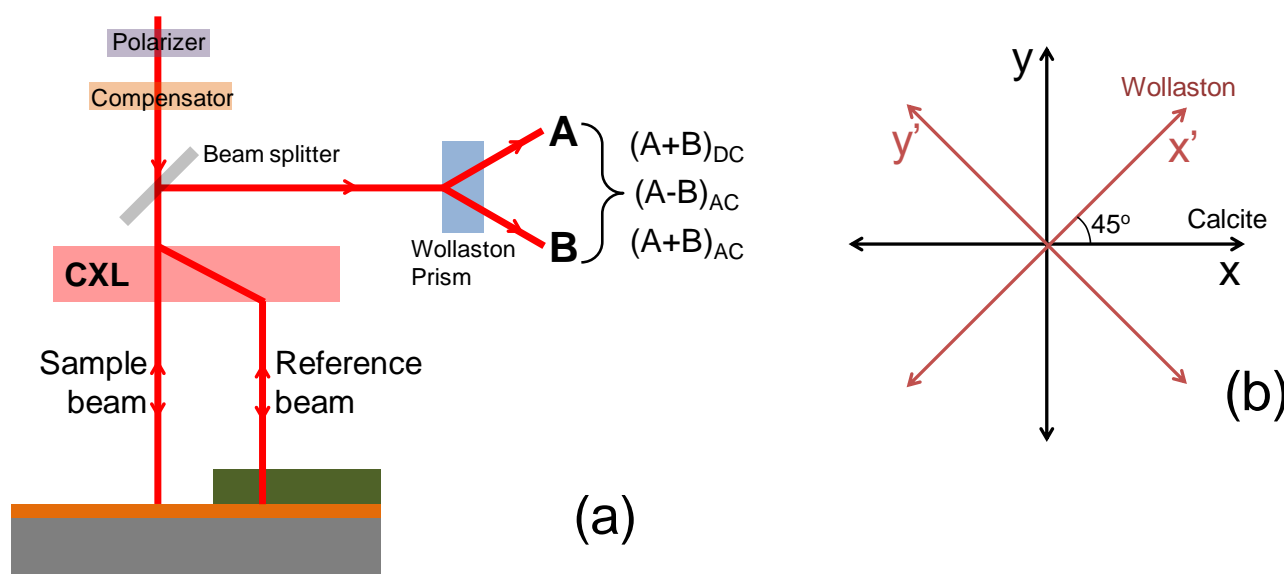

Figure S6: Beam path in SEED. a) The input polarized light is split into two beams by CXL. b) The output beam is then split by a Wollaston prism with polarization at $45^{\circ}$ with respect to the input beam polarization by $\mathrm{CXL}$.

\section{S4. The Analysis of SEED Optics}

\section{Phase modulation of the reflected light}

As shown in Figure S6(a), the polarized light is split into sample and reference beams, of the electric field given by

$$
\begin{gathered}
E_{r}=r_{o} e^{i\left(\frac{2 \pi c}{\lambda}\right) t} \\
E_{S}=s_{o} e^{i\left(\left(\frac{2 \pi c}{\lambda}\right) t+\psi\right)}
\end{gathered}
$$

where, $\omega_{0}$ is the frequency of the light; $\psi$ is a phase introduced by the compensator (and the SU8); and the polarization of the incident beam such that amplitudes (i.e., intensities) $r_{0}=S_{0}$.

Because of the AC potential at $\omega$, the path length, p.l. modulated as

$$
p . l .=\left(\int_{0}^{\kappa} \frac{d n_{+}}{d c} \delta c_{+} d x \int_{0}^{\kappa} \frac{d n_{-}}{d c} \delta c_{-} d x\right) \cos \omega t=z_{o} \cos \omega t
$$

where, $d n / d c$ is the differential refractive index, a constant, $\delta c$ is the amplitude of oscillation of the ions in the interfacial layer due to the AC potential, $\mathrm{K}$, is the penetration depth of the electric field emanating from the electrode into the solution, and $z_{0}$ is the amplitude of the path length oscillation due to the AC potential. Usually, the polarizability of the anion is significantly larger due to uncompensated electrons, thus, e $z_{0}$ is governed by concentration of anions, c- compared to cations, $\mathrm{C}_{+}$, in the solution. Assuming the system to be linear, a good assumption is that the second order signal at $2 \omega$ is very small, the amplitude of path length oscillation at $\omega$ is given by 


$$
z_{o} \approx \frac{d n_{-}}{d c} \kappa\left\langle\delta c_{-}\right\rangle
$$

where, $\langle\ldots\rangle$ is the spatial average. The resultant phase modulation due to the path length modulation, $z_{o}$ is

$$
\phi=\frac{2 \pi}{\lambda} z_{o} \cos \omega t
$$

where, $\lambda=633 \mathrm{~nm}$ is the wavelength of the He-Ne laser beam.

Thus, the optical electric fields (in Eq. (1)), after reflection, is given by

$$
\begin{aligned}
& E_{r}=r_{o} e^{i\left(\frac{2 \pi c}{\lambda}\right) t} \\
& E_{S}=r_{o} e^{i\left[\left(\frac{2 \pi c}{\lambda}\right) t+\psi+\phi\right]}
\end{aligned}
$$

Furthermore, another phase difference, $\alpha$, is introduced due to reflectivity at the electrolyte/electrode interface relative to the SU8/electrode interface. Thus, the reflected light will be

$$
\begin{aligned}
& E_{r}=r_{o} e^{i\left(\frac{2 \pi c}{\lambda}\right) t} \\
& E_{S}=R r_{o} e^{i\left[\left(\frac{2 \pi c}{\lambda}\right) t+\psi+\phi+\alpha\right]}
\end{aligned}
$$

where, $R$ is the reflectivity of the sample beam relative to the (constant) reflectivity of the reference beam. As a result, the reflectivity to the reference beam is set to 1 . From Fresnel's law, the complex reflectivity amplitude is given by

$$
\mathbb{R}=\frac{N_{1}-N_{2}}{N_{1}+N_{2}}=R e^{i \alpha}
$$

where, $N_{1}=<n_{1}-i k_{1}>$ and $N_{2}=n_{2}-i k_{2}$ are complex refractive indices of the electrolyte at the interface and electrode, respectively. Typically, the Fresnel reflectivity, $\mathrm{R}_{\mathrm{F}}$, due to $\mathrm{MB},<\mathrm{n}_{1}>\sim 1.5$ and $<\mathrm{k}_{1}>\sim 10^{-3}$; and $633 \mathrm{~nm}$ for the Au electrode, $\mathrm{n}_{2} \sim 0.21$ and $\mathrm{k}_{2} \sim$ 3.24, Eq. (7) can be approximated as,

$$
\begin{aligned}
& R=R_{F}=1-\frac{2\left\langle n_{1}\right\rangle n_{2}}{\left\langle n_{1}\right\rangle^{2}+k_{2}^{2}}=1-K^{\prime}{ }_{o} \\
& \alpha=\frac{2\left\langle n_{1}\right\rangle}{k_{2}}
\end{aligned}
$$

To derive Eq. (8) from Eq. (7) we have assumed, $\mathrm{k}_{1}<<\mathrm{k}_{2},\left(<\mathrm{n}_{1}>\right)^{2}>>\left(\mathrm{n}_{2}\right)^{2}$, and $<\mathrm{n}_{1}>\mathrm{k}_{2}$ $>>n_{2} k_{1}$. To note is that for typical values of the solution and the electrode, $K_{\circ} \circ \sim 0.05$ and $\alpha \sim 0.86$ radians.

The AC potential will also modulate the reflectivity via the oscillation of the ions in the vicinity of the electrode surface. As the Fermi level of $A u$ is lower than the solution, ${ }^{1}$ the 
interface (very close to the surface) will primarily have anions. Thus, in the vicinity of the surface, the refractive index of the solution due to the AC potential is given by,

$$
n_{s}=\left\langle n_{1 s}\right\rangle+\frac{d n_{-}}{d c}\left\langle\delta c_{s}\right\rangle \cos \omega t=\left\langle n_{1 s}\right\rangle+\delta n_{s} \cos \omega t
$$

where, constant $\left\langle\mathrm{n}_{1 \mathrm{~s}}\right\rangle$ is the (spatially averaged) effective refractive index of the solution at the Au surface, and $\left\langle\delta c_{s}\right\rangle$ is the spatially averaged amplitude of oscillation of the anions at the surface. Thus, substituting $\left\langle n_{1}\right\rangle=\left\langle n_{1 s}\right\rangle$ in Eq. 8 , the oscillation in the Fresnel reflectivity due to the $A C$ potential can be obtained as,

$$
\begin{aligned}
R_{F} & =1-\frac{2\left\langle n_{1 s}\right\rangle n_{2}}{\left\langle n_{1 s}\right\rangle^{2}+k_{2}^{2}}-2 n_{2} \frac{\left(k_{2}^{2}-\left\langle n_{1 s}\right\rangle^{2}\right)}{\left(\left\langle n_{1 s}\right\rangle^{2}+k_{2}^{2}\right)^{2}} \delta n_{s} \cos \omega t \\
& =1-K_{o}-K_{1} \delta n_{s} \cos \omega t=1-K_{o}-R_{o} \cos \omega t \\
\alpha & =\frac{2\left\langle n_{1 s}\right\rangle}{k_{2}}+\frac{2 \delta n_{s}}{k_{2}} \cos \omega t
\end{aligned}
$$

In summary, the amplitude of the reflected beam is given by, Eq. 6, where, $\phi$ is given by Eq. (4) and (3); $R_{F}$ and $\alpha$ are given by Eq. (10) and (9); and $\psi$ is a constant phase angle regulated by the compensator for nulling as discussed below.

\section{Signal Detection}

Next, we calculate the intensities, A and B at the two detectors after superposition (i.e., interference) occurs in the CXL. (This is advantageous because of the reduction in thermal noise that would occur if interference occurred in air.) To reduce thermal noise further, the CXL is mounted in an insulating foam. The output beam is then split by a Wollaston prism with polarization at $45^{\circ}$ with respect to the CXL (Figure S6(b)). As a result, the amplitudes on detectors $A$ and $B$ are

$$
\begin{aligned}
& E_{A}=\frac{1}{\sqrt{2}}\left(E_{r}+E_{S}\right) \\
& E_{B}=\frac{1}{\sqrt{2}}\left(E_{r}-E_{s}\right)
\end{aligned}
$$

where, $E_{r}$ and $E_{s}$ are given by Eq. (6) with all of the phases and reflectivity given be Eq. (4) and (10). Thus, the power (i.e., the intensity of the light), that is measured on each detector given by, $E_{A}\left(E_{A}\right)^{*}$ and $E_{B}\left(E_{B}\right)^{*}$, is

$$
\begin{aligned}
& A=\frac{1}{2}\left[r_{o}^{2}+R_{o}^{2} r_{o}^{2}+2 R_{o} r_{o}^{2} \cos (\psi+\phi+\alpha)\right] \\
& B=\frac{1}{2}\left[r_{o}^{2}+R_{o}^{2} r_{o}^{2}-2 R_{o} r_{o}^{2} \cos (\psi+\phi+\alpha)\right]
\end{aligned}
$$

The Nulling

The intensity measured on the two detectors (Eq. (12)) have both AC and DC 
components. By adding and subtracting the two differential signals (using simple hardware), the signal from Eq. (12) becomes,

$$
\begin{aligned}
& (A-B)_{A C+D C}=2 R_{o} \cos (\psi+\phi+\alpha) \\
& (A+B)_{A C+D C}=1+R_{o}^{2}
\end{aligned}
$$

The added and subtracted AC signal after amplification by the lock-in amplifier tuned at $\omega$ (see Figure 3 in main MS and Figure S6), and the DC signal is directly hardwired into the computer. As noted above, the AC oscillations are very small to maintain the linearity of the measurement. The higher harmonics (that can directly be measured from the lock-in-amplifier) of $\omega$ are at least three orders of magnitude lower. As a result, the $A C$ amplitude of $R_{F}, \phi$, and $\alpha$ are $<<1$. By collecting only the first harmonic term at $\omega$, from Eqs. (8), (10), and (13),

$$
\begin{aligned}
& \left|\frac{(A-B)_{A C}}{(A+B)_{D C}}\right|=2\left(1-K_{o}\right) \cos (\psi+\phi+\alpha) \\
& \left|\frac{(A+B)_{A C}}{(A+B)_{D C}}\right|=2\left(1-K_{O}\right) K_{1} \delta n_{s}
\end{aligned}
$$

where, the normalizing $D C$ signal, $(A+B) D C$, measured by passing the signal through a low pass filter is $\left(r_{0}\right)^{2}$. To note is that the $A C$ oscillation term, $K_{1} n_{s} \cos (\omega t)$, in Eq. (10) is dropped because it will lead to second harmonics.

Away from the redox, the compensator is adjusted such that $\psi=\pi / 2$. This is obtained by adjusting the compensator so that the baseline is minimum and flat. The important aspect of the optics is that because the two beams are always close, $<100 \mu \mathrm{m}$ apart, the thermal environment is similar, leading to very stable $\psi$ that does not require feedback during the measurement. For $\psi=\pi / 2$ and dropping higher harmonic terms and considering that the oscillation amplitudes are small, the normalized output raw signal at the computer measured in real time (see Figure 3(a)) becomes,

$$
\begin{aligned}
& \left|\frac{(A-B)_{A C}}{(A+B)_{D C}}\right|=\Delta=\frac{4 \pi}{\lambda} z_{o}+\frac{4}{k_{2}} \delta n_{S} \text { (SEED-I raw signal) } \\
& \left|\frac{(A+B)_{A C}}{(A+B)_{D C}}\right|=R=2 K_{1} \delta n_{S} \quad \text { (SEED-R raw signal) }
\end{aligned}
$$

Both $\mathrm{R}$ and $\Delta$ are dimensionless quantities that may now be compared to understand the observation made in each mode.

General remarks:

- The interferometry signal, i.e., SEED-I in Eq. (15) is a linear combination of the SEED-I and SEED-R signals, while SEED-R measures near-surface phenomena exclusively.

- There is some amount of amplification in the SEED-I signal because $z_{0}$ 
includes oscillations extending "deep" into the solution, while the signal is from the near-surface process for reflectivity.

- SEED-I should always yield a signal, while the signal may be weak for conventional redox processes in SEED-R where the redox ions are in the solution.

- SEED-R may be more sensitive to redox ions confined to the surface.

- In principle, only one beam is needed for SEED-R.

- Both $\langle\delta c\rangle$ (in Eq. (3)) and $\left\langle\delta c_{s}>\right.$ (in Eq. 9) are linearly proportional to the (local) redox current, where the proportional constant will have to be calibrated.

\section{S5. Figures $\mathrm{S} 7$ to $\mathrm{S} 16$}

Following are nine illustrations that aid the discussion in the main manuscript.

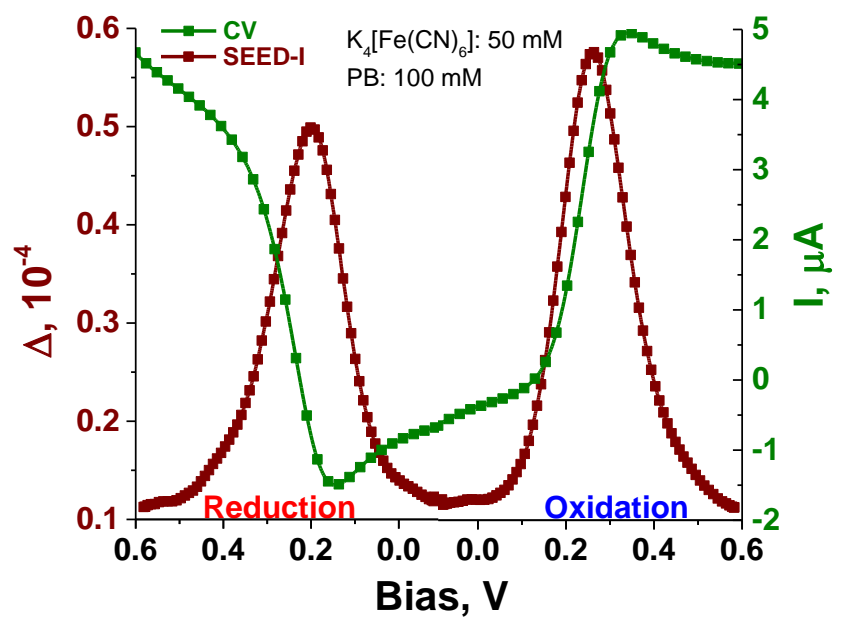

Figure S7: Using the setup in Figure 2, the CV and SEED-I were concomitantly measured for $50 \mathrm{mM} \mathrm{K} 4\left[\mathrm{Fe}(\mathrm{CN})_{6}\right]$ in $100 \mathrm{mM} \mathrm{PB}$ at a ramp rate of, $\mathrm{s}=0.05 \mathrm{~V} / \mathrm{s}$. The SEED-R signal was from the $6-\mu \mathrm{m}$ spot of area $2.83 \times 10^{-7} \mathrm{~cm}^{2}$; and the $\mathrm{CV}$ signal was from all of the 72 microwells with a combined area of $1.41 \times 10^{-3} \mathrm{~cm}^{2}$. Thus, the area of SEED-I was $5 \times 10^{3}$-fold smaller than CV. 


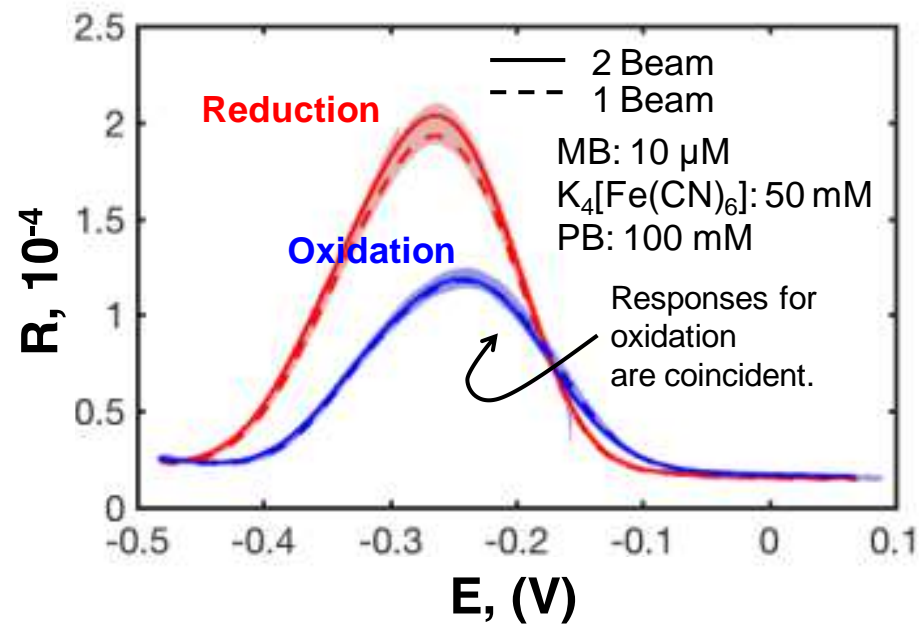

Figure S8: Comparison between SEED-R signal with two beam (i.e., Figure 2) and clipping the reference beam after the CXL.
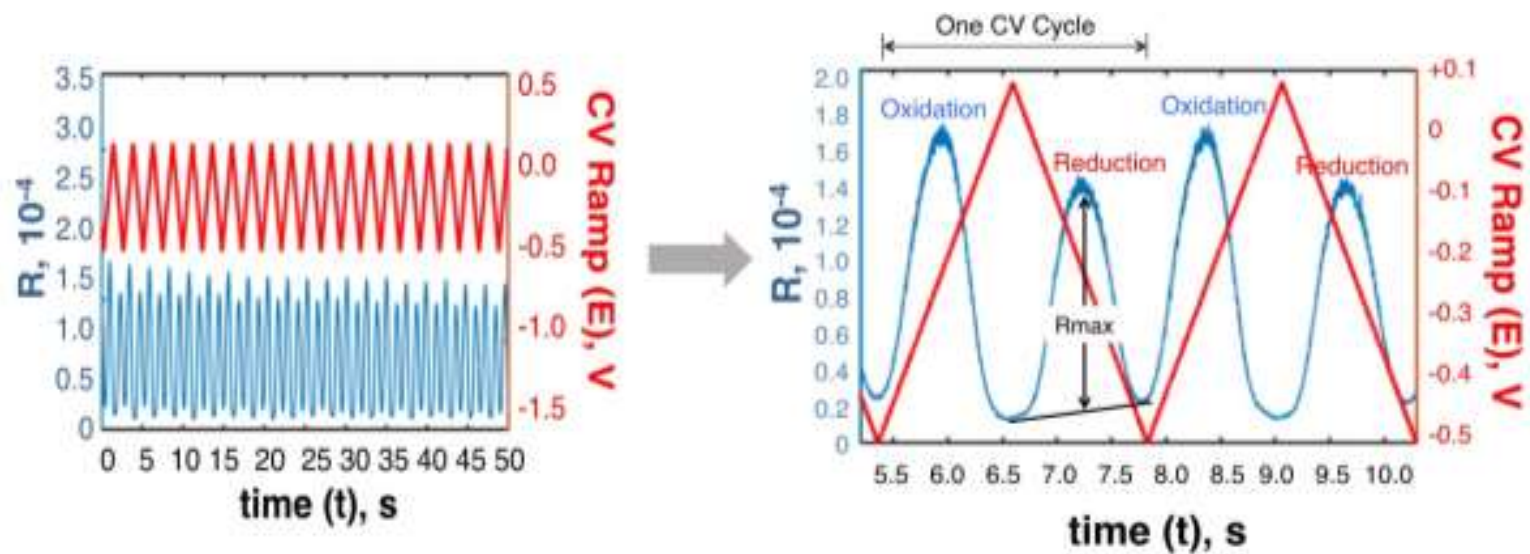

Figure S9: Raw data showing peaks for $\mathrm{MB}^{+} / \mathrm{LMB}$ redox

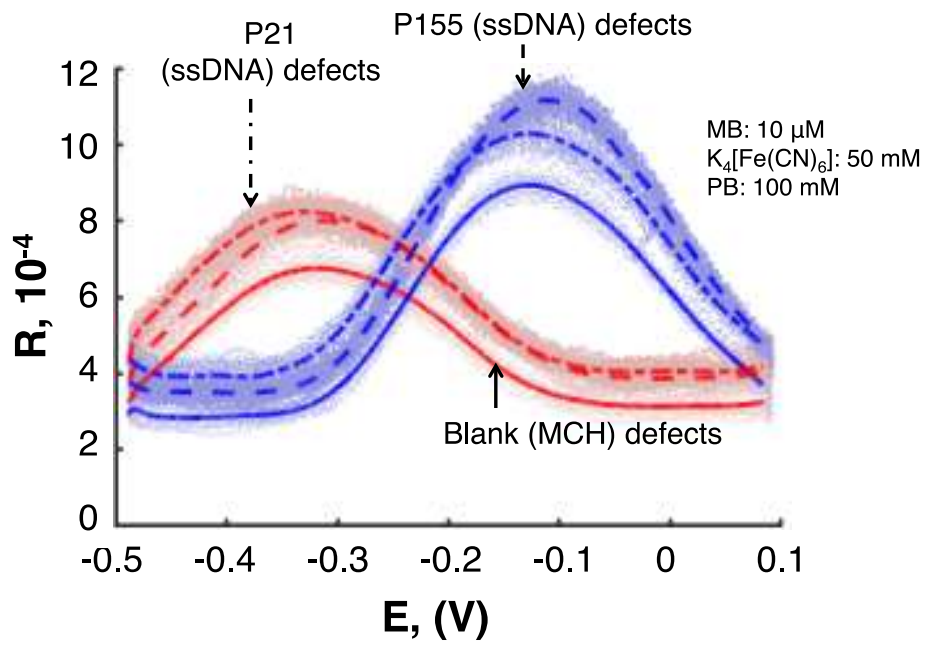

Figure S10: Typical oxidation (blue) and reduction (red) SEED-R signal on the control electrode for the three microwells (blank, P155, and P21) when the backfilling was not complete. All of the spots on each microwell showed a positive signal. 


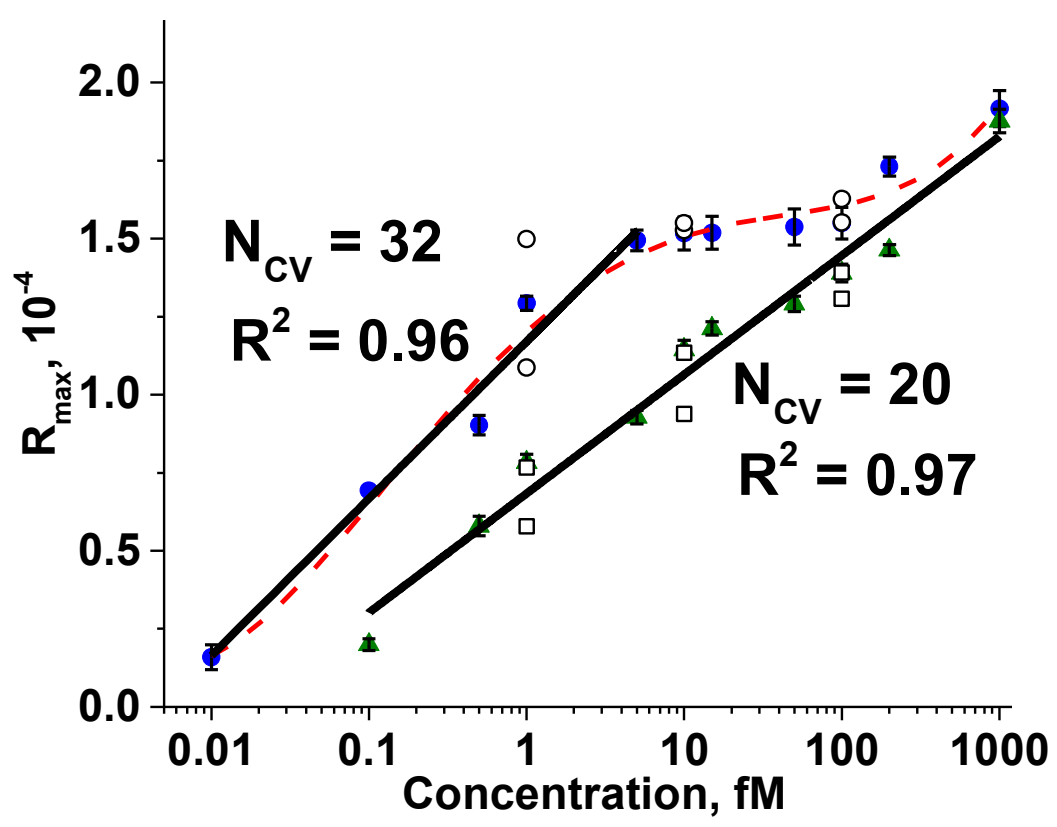

Figure S11: The SEED-R signal as a function of target concentration, [c]. The probe and target were P155 and miR-155, respectively. The fitness, $\mathrm{R}^{2}>0.96$, indicated excellent correlation over a dynamic range of five orders of magnitude. The data was reproduced from our previous publication. ${ }^{2}$

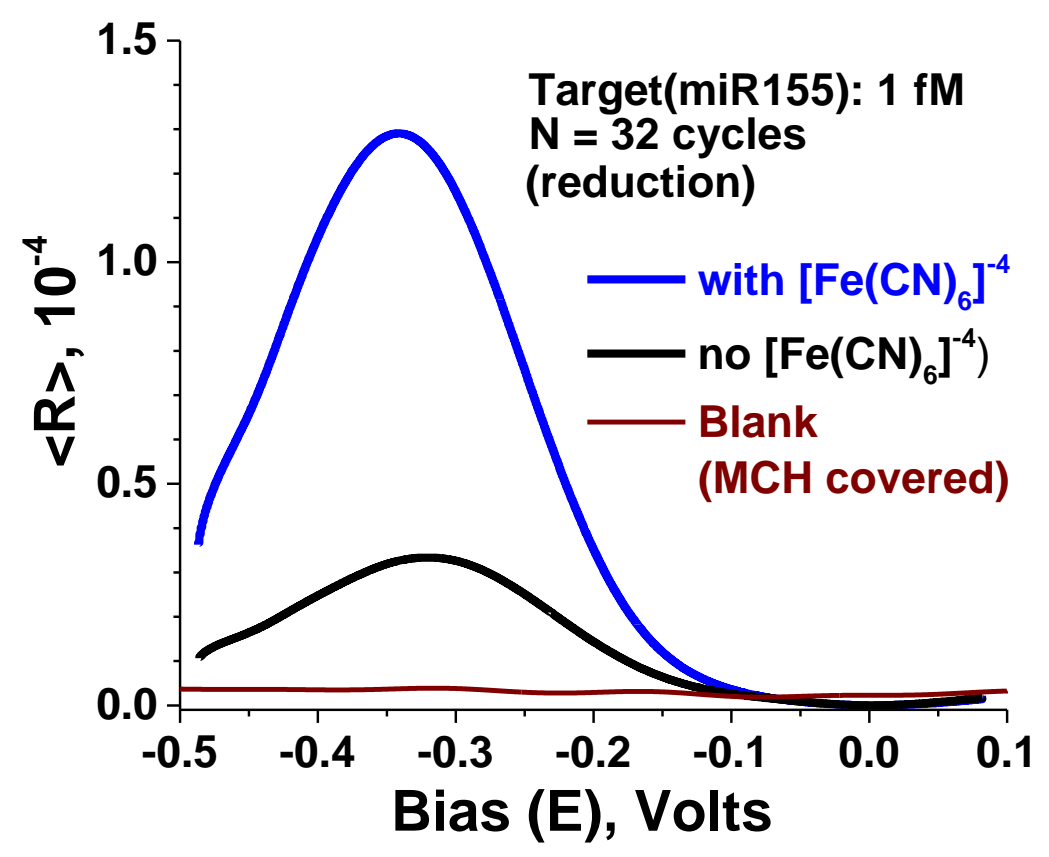

Figure S12: Effect of including auxilary redox ion, $\left[\mathrm{Fe}(\mathrm{CN})_{6}\right]^{4-}$ on EREB. 


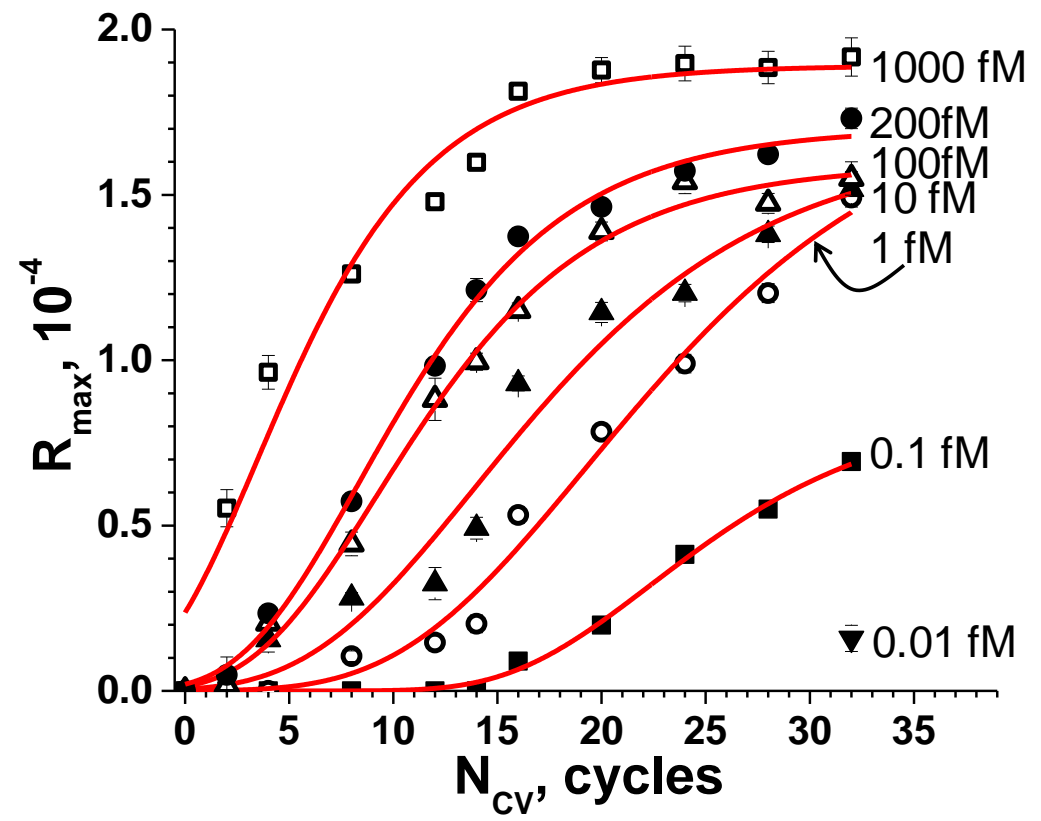

Figure S13: Effect of EREB cycles, Ncv on EREB for [c] ranging from $10^{-2}$ to $10^{3} \mathrm{fM}$
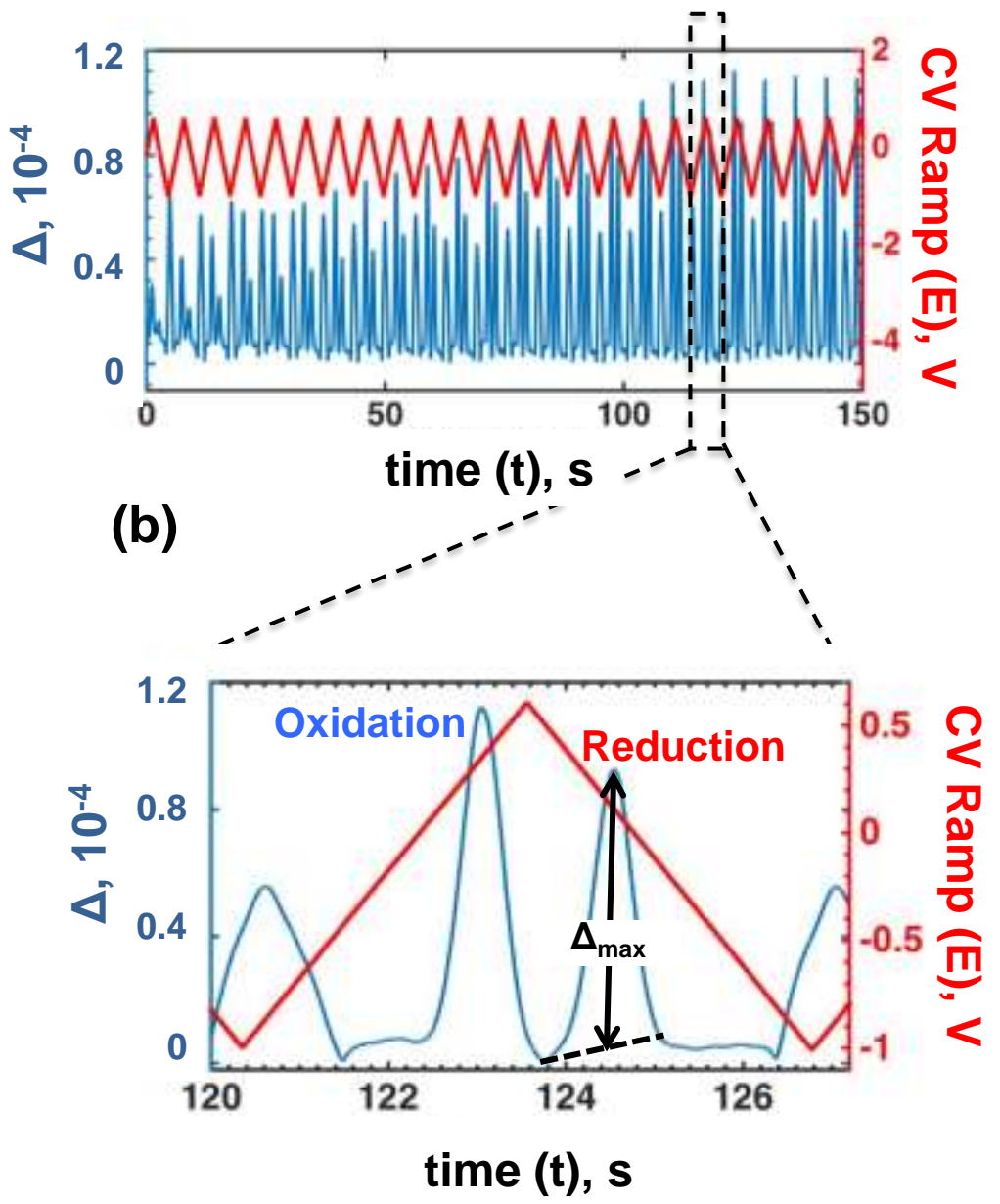

Figure S14: SEED-I raw data during $\mathrm{MCH} / \mathrm{ssDNA}$ stripping showing the increase in $\mathrm{Fe}\left(\mathrm{CN}_{6}\right)^{3-14-}$ redox peaks. The zoom-in panel shows one CV cycle with oxidation and a reduction signal peak of $\mathrm{Fe}\left(\mathrm{CN}_{6}\right)^{3-4-}$. 


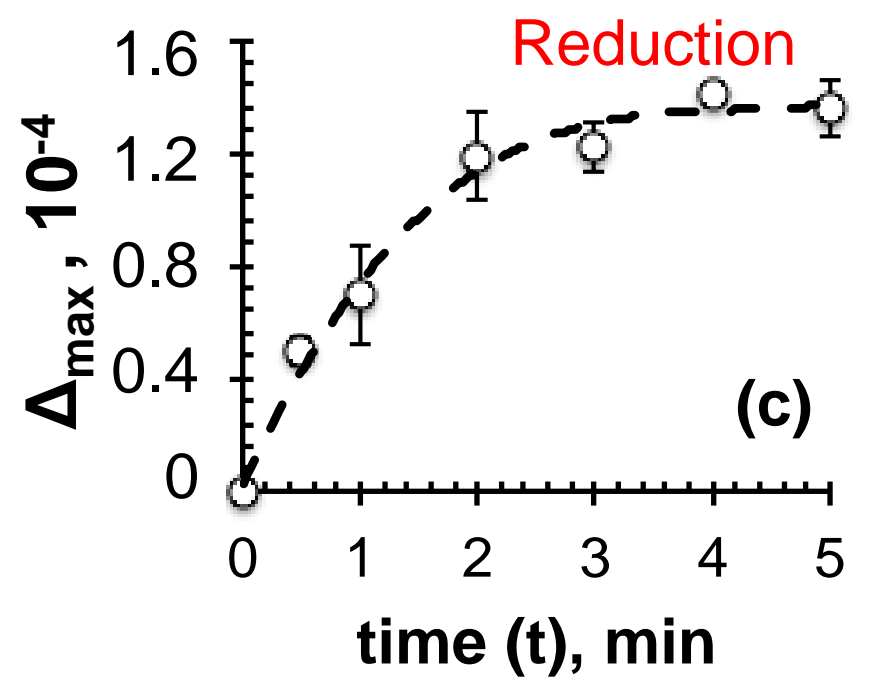

Figure S15: The $\Delta_{\max }$ of $\mathrm{Fe}\left(\mathrm{CN}_{6}\right)^{3-14-}$ redox during stripping.

a)

$\left[\mathrm{Fe}(\mathrm{CN})_{6}\right]^{4-1 / 3-r e d o x ~ s i g n a l ~ o n ~ a ~}$ bare Au.

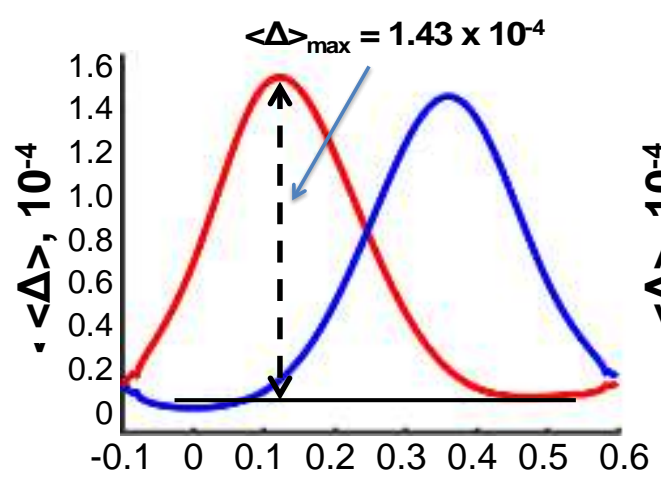

Bias (E), V b)

$\left[\mathrm{Fe}(\mathrm{CN})_{6}\right]^{4-1 / 3-r e d o x ~ s i g n a l ~ a f t e r ~}$ stripping $\mathrm{MCH}$ and probe DNA from $\mathrm{Au}$.

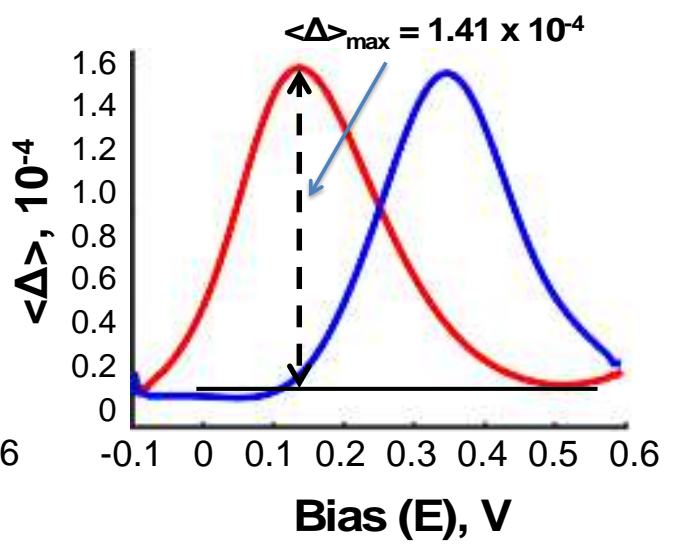

Figure S16: Comparison of SEED-I redox signal for $50 \mathrm{mM} \mathrm{K}_{4}\left[\mathrm{Fe}(\mathrm{CN})_{6}\right]$ in $100 \mathrm{mM}$ PB on (a) pristine Au after cleaning with piranha solution and (b) a Au electrode after the $\mathrm{MCH}$ was electrochemically removed. The nominally similar signal indicated that the removal of $\mathrm{MCH}$ after 5 min was complete.

\section{S6. Fluorescence Analysis for Probe Coverage}

The P155 probes tagged with Cy-3 fluorescent dye at 3' (Cy3-P155) were immobilized on the $\mathrm{Au}$ electrode of a chip. The electrode was a $0.08 \mathrm{~cm} \times 0.8 \mathrm{~cm}$ rectangle patterned by lithography. A fluorescence emission spectrum was recorded at an excitation wavelength of $550 \mathrm{~nm}$ (F-4500 Fluorescence Spectrophotometer, Hitachi) (Figure $\mathrm{S} 17(\mathrm{a})$ ). The peaks were resolved by fitting three Gaussian curves at the 
wavelengths noted in Table S1. The Gaussian peak at the wavelength of $551.7 \mathrm{~nm}$ corresponds to Au (Peak 1), $574.0 \mathrm{~nm}$ to Cy-3 (Peak 3), and $641.6 \mathrm{~nm}$ to P155 (Peak 2).

To avoid the quenching Cy3 dye, the gold surface was passivated with dielectric $\mathrm{MCH}$. As shown in Figure $\mathrm{S} 17(\mathrm{~b}), 1.51 \times 10^{10}$ Cy3-P155 molecules $/ \mathrm{cm}^{2}$ showed a peak at $574.0 \mathrm{~nm}$ for Cy3 with negligible height ( 0.01 a.u.), which improved by fourfold $(0.42$ a.u.) after $\mathrm{MCH}$ backfilling.

The deconvoluted Gaussian fluorescence curve, IF, was obtained for films with a known amount of Cy3-tagged probe molecules deposited on a $6.4 \times 10^{2} \mathrm{~cm}^{2}$ Au surface (Figure S17(c)).

A calibration curve was obtained by measuring peak of $I_{F}$ as a function of known amount of molecules deposited on $\mathrm{MCH}$ passivated Au surface of same area (Figure $\mathrm{S} 17(\mathrm{~d})$ ). From the fluorescence of immobilized probes (Figure S17(a)) and the calibration curve (Figure S17(d)), the probe coverage, $\mathrm{NP}$, was estimated to be $2.9 \pm 0.4$ $\times 10^{12}$ molecules $/ \mathrm{cm}^{2}$. The error was based on five chips. The coverage measured by fluorescence and qPCR was consistent, indicating that the coverage of probe ssDNA was uniform in the $50-\mu \mathrm{m}$ microwell.
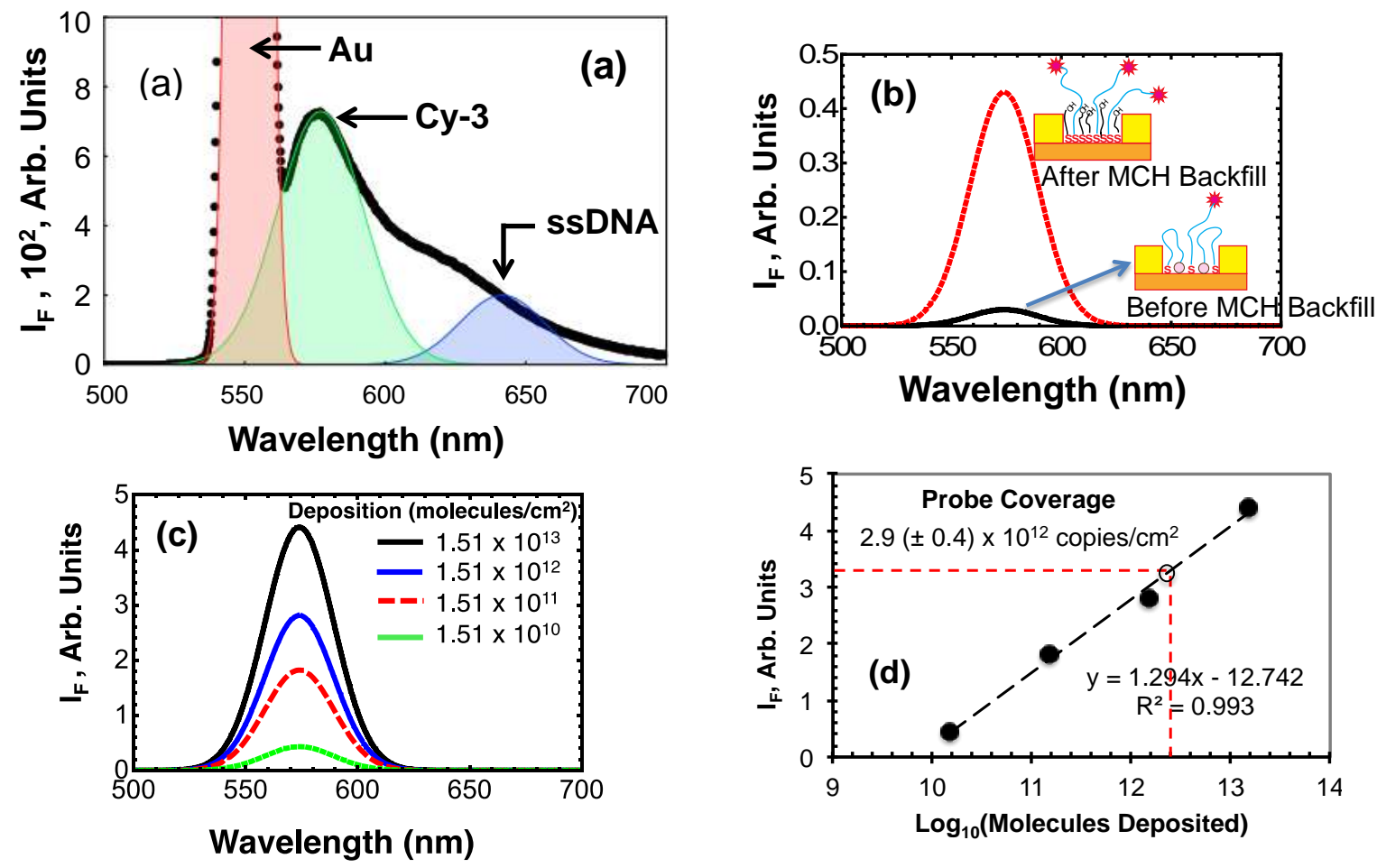

Figure S17: (a) Fluorescence emission spectrum of Cy-3-tagged ssDNA on a Au surface excited at $550 \mathrm{~nm}$. (b) Gaussian peaks for the Cy3 fluorescence before and after MCH backfilling for $1.51 \times 10^{10}$ Cy3-P155/ $\mathrm{cm}^{2}$. (c) Fitted gaussian fluorescence peaks from different amounts of Cy-3-Ibled ssDNA probe film. (d) Calibration curve showing linear fit of $\log _{10}$ (probe copy number, i.e.. coveraqe) as a function of $\mathrm{IF}_{\mathrm{F}}$. 
Table S1. Gaussian parameters obtained from fitting the experimental data in Figure S17.

\begin{tabular}{|l|c|c|c|}
\hline \multirow{2}{*}{} & \multicolumn{3}{|c|}{ Peak Number } \\
\cline { 2 - 4 } & $\mathbf{1}$ & $\mathbf{2}$ & $\mathbf{3}$ \\
\hline K $(\mathrm{nm})$ & $4.4 \pm 0.01$ & $15.4 \pm 0.11$ & $15.0 \pm 0.13$ \\
\hline Lambda $(\mathrm{nm})$ & $551.7 \pm 0.01$ & $574.0 \pm 0.04$ & $641.6 \pm 0.13$ \\
\hline
\end{tabular}

\section{S7. Differential Pulse Voltammetry (DPV)}

The chip was fabricated similar to that described in Section S1. The EREB was performed at $\mathrm{N}_{\mathrm{cv}}=5$ cycles and target concentration of $1 \mathrm{pM}$. The electrode was subsequently backfilled. Among the two chips fabricated, the $\mathrm{R}_{\max }$ for MB reduction on electrodes with good $\mathrm{MCH}$ backfill averaged at $\sim 1.5 \times 10^{-5}$ with no redox signal for $\left[\mathrm{Fe}(\mathrm{CN})_{6}\right]^{4-/ 3}$. On the same chip, two electrodes with accidental defects at a few spots leading to poor backfill showed a twofold larger $R_{\max }$ and a redox signal for $\left[\mathrm{Fe}(\mathrm{CN})_{6}\right]^{4-/ 3}$. A typical SEED signal for poor MCH backfill is shown in Figure S18.

To circumvent the overriding large capacitance current observed in CV, redox is measured by differential pulse voltammetry (DPV). DPV was performed using Autolab NOVA 2.1 on all of the electrodes with the following conditions: step $=0.005 \mathrm{~V}$, modulation amplitude $=0.02 \mathrm{~V}$, modulation time $=0.005 \mathrm{~s}$; and interval time $=0.5 \mathrm{~s}$. Consistent with the SEED results, the current due to MB reduction on a poorly backfilled electrode was significantly larger.

The DPV for $10 \mu \mathrm{M} \mathrm{MB}$ and $50 \mathrm{mM} \mathrm{K}_{4}\left[\mathrm{Fe}(\mathrm{CN})_{6}\right]$ in $100 \mathrm{mM} \mathrm{PB}(\mathrm{pH} 7.6)$ showed a strong peak due to $\mathrm{MB}$ reduction (Figure S19).

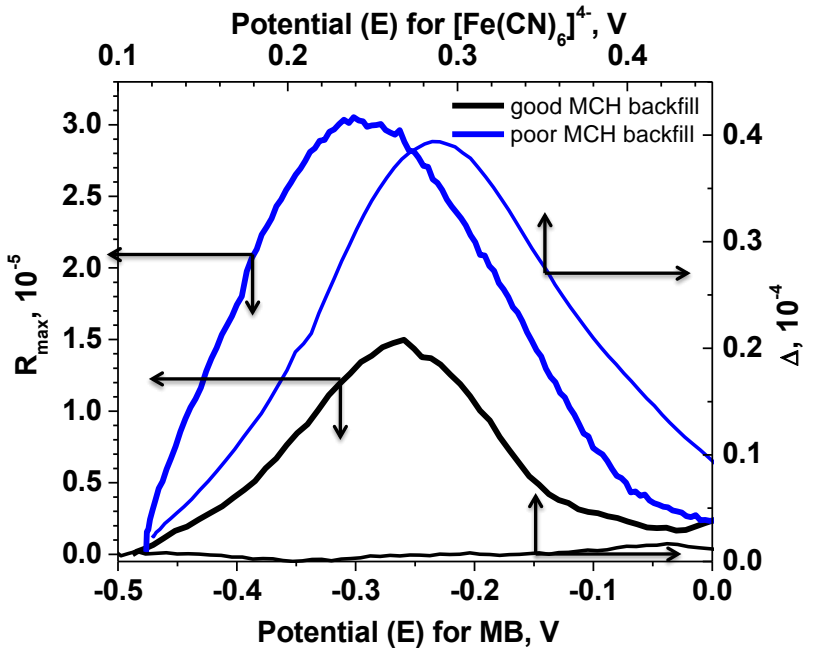

Figure S18: SEED signal comparing two spots on the same electrode with good and poor backfilling of $\mathrm{MCH}$. Reduction of $\mathrm{MB}$ and $\mathrm{K}_{4}\left[\mathrm{Fe}(\mathrm{CN})_{6}\right]$ are shown for corresponding spots. 


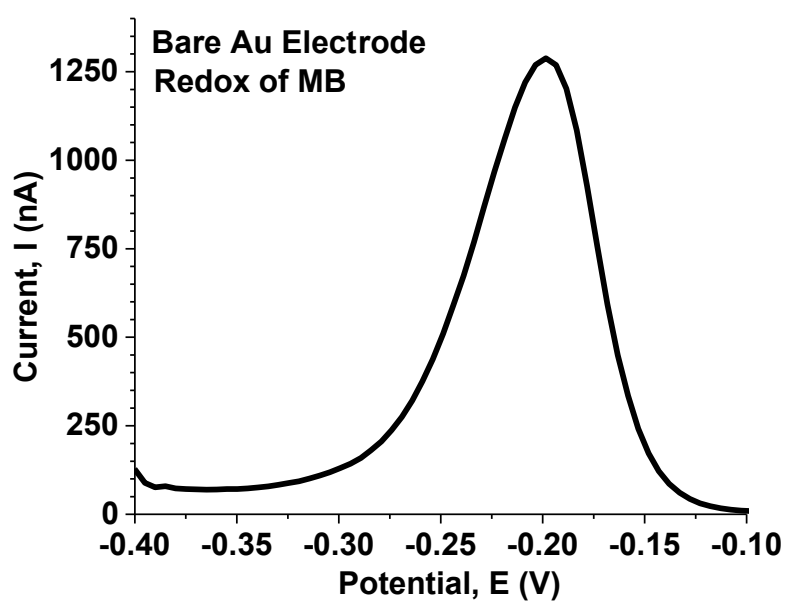

Figure S19: DPV showing the $\mathrm{MB}$ reduction on pristine Au electrode surface.

\section{References}

1. Yu, C. C.; Lee, S. W.; Ong, J.; Moore, D.; Saraf, R. F. Single Electron Transistor in Aqueous Media. Adv. Mater. 2013, 25, 3079-3084.

2. Raghunath, S.; Prasad, A.; Tevatia, R.; Gunther, J. R.; Roy, S.; Krishnan, S.; Saraf, R. F. Quantitative Electrochemical DNA Microarray on a Monolith Electrode with Ten Attomolar Sensitivity, 100\% Specificity, and Zero Background. Chemelectrochem 2018, 5, 429-433. 